\title{
Autonomia-Relacionada como Tendência do Desenvolvimento do Self: Novas Evidências em um Contexto Brasileiro
}

\author{
Maria Lucia Seidl-de-Moura ${ }^{1}$ \\ Dandara de Oliveira Ramos \\ Universidade do Estado do Rio de Janeiro \\ Luciana Fontes Pessôa \\ Pontifícia Universidade Católica do Rio de Janeiro \\ Rafael Vera Cruz de Carvalho \\ Tânia Abreu da Silva Victor \\ Deise Maria Leal Fernandes Mendes \\ Universidade do Estado do Rio de Janeiro
}

\begin{abstract}
RESUMO - Estudos brasileiros sobre as trajetórias de desenvolvimento do self, em sua maioria com mães de crianças pequenas, indicam predominância do modelo de autonomia-relacionada (AR). Visando ampliar a investigação dessa tendência, foram realizados dois estudos: (a) com avós(ôs), mães, pais e filhos (16-25 anos) e (b) quatro grupos de cuidadoras de crianças com até um ano, com níveis de escolaridade diversos, selecionados não aleatoriamente. Observamos predomínio de características de self AR para todos os participantes. O efeito da escolaridade sobre autonomia foi confirmado para os pais, a AR de pais e filhos (estudo 1), e de mães (estudo 2). Mães criadas no Rio de Janeiro (capital) apresentaram maiores escores de autonomia. A consistência familiar de modelo de self autônomo-relacionado foi evidenciada.
\end{abstract}

Palavras-chave: autonomia-relacionada, gerações familiares, cuidadoras de crianças, modelos do desenvolvimento do self

\section{Related Autonomy as a Tendency in Development of the Self: New Evidence from a Brazilian Context}

\begin{abstract}
Studies in Brazil on developmental trajectories of the self, mostly with mothers of young children, indicate a prevalence of the related autonomy model (RA). Aiming to broaden the investigation of this trend, two studies were realized: (a) with grandparents, mothers, fathers and their sons and daughters (16-25 years old), and (b) with four groups of caretakers of children up to one year old, with diverse educational levels, non-randomly selected. We observed predominance of RA self for all participants. The effect of school level over autonomy was confirmed for fathers, RA of fathers and sons and daughters (study 1), and of mothers (study 2). Mothers who were raised in Rio de Janeiro (state capital) had higher autonomy scores. The results support the family consistency of the related-autonomy model of development of the self.
\end{abstract}

Keywords: related autonomy, family generations, caregivers of children, self development models

O desenvolvimento da autonomia, da capacidade de agir no mundo, bem como da necessidade de nos vermos como separados dos outros, é fundamental para nossa possibilidade de sobreviver. Por outro lado, somos membros de uma espécie com uma organização complexa e a relação com nossos coespecíficos também é essencial para a nossa sobrevivência tanto física como psicológica (Keller, 2007). Algumas perspectivas psicológicas priorizam a autonomia, a separação e a independência para o desenvolvimento emocional saudável. No entanto, tanto a autonomia em seu sentido de agência como a capacidade de estabelecer relações sociais são tendências universais e desenvolvidas na ontogênese (Greenfield, Keller, Fuligni, \& Maynard, 2003; Kağitçibaşi, 2007, Keller, 2007). Segundo essas autoras, pesquisas empíricas têm demonstrado a grande variedade de formas que o "desenvolvimento sadio" pode

1 Endereço para correspondência: Rua Fritz Feigl, 465, Jacarepaguá, Rio de Janeiro, RJ, Brasil. CEP: 22.750-600.E-mail: mlseidl@gmail.com assumir em diferentes culturas, envolvendo um equilíbrio entre a aquisição da autonomia e a capacidade de estabelecer relações com os outros e ser próximo a eles, com variações na extensão em que cada dimensão é enfatizada.

Greenfield et al. (2003) propõem que existem tarefas universais de desenvolvimento: formação de relacionamentos, aquisição de conhecimento e equilíbrio entre autonomia e relação. Para essas autoras, essas tarefas são relevantes em momentos diversos da ontogênese, a primeira desde o nascimento, a segunda na infância inicial e a terceira na adolescência. São construídas ao longo do ciclo vital. Desenvolvemos relações interpessoais, construímos conhecimento e desenvolvemos um self em que a autonomia e a relação com os outros são diferencialmente valorizadas ao longo de nossa vida e em contextos culturais específicos.

As trajetórias de desenvolvimento seguidas na organização dessas tarefas, ou modelos de self, têm sido descritos por diversos autores, como Keller $(2007,2012)$ e Kağitçibaşi (1996, 2007, 2012). Embora com diferenças em 
suas perspectivas teóricas (para uma comparação detalhada, vide Carvalho, Seidl-de-Moura, Vieira, \& Martins, 2014), as autoras concordam com a presença de tendências a balancear entre a autonomia e a relação em medidas variadas em diferentes contextos. Kağitçibaşi (2005) propõe dois eixos: um de agência, que varia da autonomia à heteronomia, e outro de distância interpessoal, que varia da proximidade à separação. A combinação dos dois leva a quatro modelos prototípicos de desenvolvimento do self.

Priorizando a autonomia no eixo de agência e a separação no eixo de distância interpessoal, estaria o primeiro modelo de self: o autônomo-separado. Este é prevalente do Ocidente, em grupos urbanos, escolarizados, pós-industrializados, com orientação para a independência intergeracional, valorização da autoconfiança e da autovalorização do indivíduo, com menor desenvolvimento da proximidade interpessoal. Corresponderia ao modelo de família de independência. O segundo modelo de self, por sua vez, com ênfase na heteronomia e na separação, é característico de sociedades patriarcais, em que tanto a relação interpessoal se mostra distante, quanto não se valoriza a ação autônoma do indivíduo. O terceiro modelo caracteriza-se pela tendência ao desenvolvimento de selves heterônomo-relacionados, característicos de culturas não ocidentais, baseadas na subsistência, com menos desenvolvimento socioeconômico, em geral, em áreas rurais. Nesse modelo, é valorizada a proximidade interpessoal, a obediência e a lealdade familiar, levando à interdependência material e emocional. Seria ligado ao modelo de família de total interdependência. Finalmente, o quarto modelo de self, o autônomorelacionado, é característico de contextos tradicionalmente interdependentes que passaram por processos de urbanização, com desenvolvimento econômico e aumento de oportunidades educacionais. Nesse modelo, espera-se que as pessoas desenvolvam autonomia, tomem decisões e ajam por conta própria, mas que também se mantenham próximas nos relacionamentos, dada a valorização da interdependência emocional. É visto como associado a um modelo de família de interdependência emocional e psicológica.

Desses modelos de desenvolvimento do self, focaliza-se particularmente neste trabalho, o autônomo-relacionado, que representa certo equilíbrio entre a valorização da autonomia e da relação. Kağitçibaşi (2012) argumenta que esse modelo pode ser considerado uma meta de desenvolvimento saudável para o mundo globalizado contemporâneo. Argumenta que ele une a valorização da tomada de decisão e atuação de modo autônomo pelo indivíduo à valorização da habilidade de se relacionar e cooperar com os outros. Desse modo, o self autônomo-relacionado seria um modelo ajustado, tanto às necessidades da espécie, quanto às necessidades contemporâneas.

A maioria dos estudos sobre esses modelos tem sido realizada em contextos prototípicos e com grupos de mães dos mesmos, como, por exemplo, na Alemanha, Costa Rica, México, Turquia, Índia, entre outros (Keller et al., 2007; Keller et al., 2005). Estudos brasileiros realizados na última década (Seidl-de-Moura, Carvalho, \& Vieira, 2013) têm complementado esses dados com explorações que vão além de utilizar apenas um grupo de um país, considerando-o prototípico. Buscam, em contraste, semelhanças e variações em diferentes contextos do Brasil (cidades, interior, diferentes regiões, etc.).

Investigações foram feitas focalizando as metas de socialização (Ruela, 2006; Seidl-de-Moura et al., 2008; Seidlde-Moura et al., 2009; Vieira, Seidl-de-Moura, Macarini, et al., 2010), as crenças maternas sobre práticas de cuidado (Vieira, Seidl-de-Moura, Lordelo, et al., 2010), a descrição das mães sobre seus filhos (Seidl-de-Moura, Mendes, et al., 2013) e as trajetórias de desenvolvimento do self no país (vide Seidl-de-Moura, Carvalho et al., 2013).

O que esse conjunto de estudos e suas evidências têm, em geral, indicado é que, entre as mães brasileiras de diferentes contextos sociais (16 cidades), há a valorização de um modelo de autonomia-relacionada, que assume formas próprias dependendo de variáveis sociodemográficas como o nível educacional, o lugar de criação e a densidade demográfica. Perceberam-se diferenças entre crenças de mães criadas em capitais e cidades do interior e o efeito significativo do tamanho da população sobre essas crenças. Uma das limitações dessa literatura (vide Seidl-de-Moura, Carvalho et al., 2013, para uma discussão) é que, com exceção de Ruela (2006), nos demais estudos não foram considerados outros membros da família, como pais e avós. Foram focalizadas mães de crianças pequenas (não mais do que seis anos de idade), porém outros cuidadores membros ou não da família, não foram incluídos. Questiona-se se a mesma tendência a privilegiar um modelo de autonomia-relacionada seria observada caso o foco dos estudos fosse ampliado.

Para Kağitçibaşi (2007), como foi visto, é na família, e por meio da transmissão geracional, que se constrói a autonomia e relação com os outros, uma vez que o sujeito constrói sua autonomia por meio de vínculos de dependência familiar. Cabe investigar, portanto, a valorização de autonomia e/ou da relação com os outros na família, incluindo diferentes gerações.

No decorrer dos anos, algumas modificações, principalmente com a inserção da mulher no mercado de trabalho e a modificação na rede de apoio em relação aos cuidados infantis, tornaram-se bem marcantes. Um exemplo disso é que o cuidado de crianças pequenas, que antes era quase que exclusivamente delegado à figura materna, atualmente, tem sido assumido, principalmente em famílias de nível socioeconômico médio e alto, por creches, babás ou avós maternas (Rapoport \& Piccinini, 2004). A partir desse novo cenário, ao se pensar no desenvolvimento inserido em contextos específicos e em possíveis trajetórias que assume, é necessário considerar as crenças dos diferentes cuidadores, como no caso deste estudo, as professoras de creche, babás e avós.

Em relação a babás, os estudos são escassos. Greenfield, Flores, Davis e Salinkham (2008) compararam mães americanas (empregadoras) e babás imigrantes latinas e americanas em suas crenças sobre práticas e metas de desenvolvimento. As autoras hipotetizaram diferenças entre as mães americanas e as babás e a influência de variáveis sociodemográficas nessas orientações culturais independentemente da origem étnica. As hipóteses foram confirmadas.

Crenças de professoras de creche brasileiras foram estudadas por Friedlmeier, Schäfermeier, Vasconcellos e 
Trommsdorff (2008) em um estudo transcultural com mães e professoras de creche alemãs e brasileiras. As professoras alemãs apresentaram resultados significativamente mais altos na valorização da independência do que as brasileiras e o reverso foi observado para a relação com os outros, ou interdependência. Mães e professoras de cada um dos dois grupos culturais não diferiram em sua orientação, embora as professoras brasileiras tenham apresentado tendência significativamente maior a valorizar interdependência do que as mães. Esse último resultado permite hipotetizar que a mesma tendência observada nos estudos brasileiros (Seidl-de-Moura, Carvalho et al., 2013) estará presente nesta investigação, qual seja a valorização de um modelo de autonomia-relacionada.

Ressalta-se que, em estudos brasileiros (Seidl-de-Moura, Carvalho et al., 2013), outros membros da família e outros cuidadores além de mães não foram incluídos. No presente trabalho, o foco foi ampliado em dois sentidos. No Estudo 1, a família é focalizada em três gerações (avós, mãe, pai e filho ou filha) e é considerada uma etapa do desenvolvimento do filho não considerada em estudos anteriores, a saber, a da idade superior a 16 anos. Por outro lado, no Estudo 2, outros tipos de cuidadores de crianças menores do que um ano são incluídos (babás, avós e professoras de creche), além da mãe. O objetivo geral desses dois estudos é, então, investigar a valorização de autonomia e da relação com os outros nos modelos de self de três gerações das famílias estudadas e de diferentes cuidadores em fases iniciais do desenvolvimento. Com isso, visa-se contribuir para a compreensão do desenvolvimento em contextos brasileiros, focalizando o Rio de Janeiro e complementando o estudo de Seidl-de-Moura et al. (2009).

Esta investigação é exploratória, mas pode trazer ainda uma contribuição à compreensão do conceito de autonomiarelacionada e das variáveis a ele associadas e ao conhecimento de modelos do self de indivíduos (cuidadores de crianças e jovens e dos próprios jovens) em contextos brasileiros. Algumas perguntas para refletir são: há uma consistência de crenças e modelos do desenvolvimento do self em gerações da mesma família? A tendência que foi observada em estudos com mulheres, mães de crianças pequenas, será observada em homens (pais) da mesma geração e em avós que são de geração diferente? Diferentes cuidadores do mesmo contexto cultural compartilham modelos de desenvolvimento do self ou há variações entre eles? Que variáveis afetam o modelo de autonomia-relacionada e que relação apresentam entre si? Essas questões não são totalmente respondidas com os dois estudos aqui apresentados, mas estes, acredita-se, trazem um avanço em nosso conhecimento sobre o tema, relevante para quem pensa que desenvolvimento é um processo que se dá em contextos específicos.

\section{Método}

Para a realização do presente estudo, foram obtidos dados de dois grupos distintos. Do Estudo 1, participaram membros de 107 famílias (pai, mãe, um filho ou filha com idade superior a 16 anos completos) residentes na cidade do Rio de Janeiro. Do Estudo 2, participaram 60 duplas constituídas por mães, avós, babás e professoras de creche (20 duplas de mães e avós; 20 duplas de mães e babás; 20 duplas de mães e professoras de creche) que compartilhavam os cuidados de bebês com idade de até um ano completo.

A Tabela 1 apresenta as características sociodemográficas dos participantes de ambos os estudos.

\section{Considerações Éticas}

O projeto Autonomia e Interdependência em Famílias do Rio de Janeiro foi submetido ao comitê de ética da Universidade do Estado do Rio de Janeiro e aprovado sob o número de protocolo 010.3.2010.

\section{Instrumentos}

Para a coleta de dados, em ambos os estudos, foram utilizadas versões idênticas dos seguintes instrumentos: (a)

Tabela 1. Características sociodemográficas dos participantes dos Estudos 1 e 2

\begin{tabular}{|c|c|c|c|c|c|c|c|c|c|}
\hline & \multirow{2}{*}{$\mathbf{N}$} & \multirow{2}{*}{$\begin{array}{l}\text { Média de } \\
\text { Idade (DP) }\end{array}$} & \multicolumn{3}{|c|}{ Escolaridade (\%) } & \multicolumn{2}{|c|}{$\begin{array}{c}\text { Local de } \\
\text { criação (\%) }\end{array}$} & \multicolumn{2}{|c|}{ Sexo (\%) } \\
\hline & & & $\begin{array}{l}\text { Até o Ens. Médio } \\
\text { incompleto }\end{array}$ & $\begin{array}{l}\text { Ens. Médio } \\
\text { completo }\end{array}$ & $\begin{array}{c}\text { Acima do Ens. } \\
\text { Superior incompleto }\end{array}$ & Capital & $\begin{array}{l}\text { Fora da } \\
\text { Capital }\end{array}$ & Fem & Masc \\
\hline \multicolumn{10}{|l|}{ Estudo 1} \\
\hline Avós/Avôs & 54 & $72,37(6,66)$ & 74,1 & 13 & 13 & 44 & 54 & 75,9 & 24,1 \\
\hline Pais & 107 & $51,57(6,55)$ & 31,8 & 19,6 & 48,6 & 68,2 & 31,8 & - & - \\
\hline Mães & 107 & $48,3(5,54)$ & 24,3 & 18,7 & 57 & 61,7 & 38,3 & - & - \\
\hline Filhos & 107 & $20,94(2,42)$ & 8,4 & 13,1 & 78,5 & 67,3 & 32,7 & 68,2 & 31,2 \\
\hline \multicolumn{10}{|l|}{ Estudo 2} \\
\hline Mães & 60 & $31,92(4,53)$ & 5 & 10 & 85 & 71,7 & 28,3 & - & - \\
\hline Babás & 20 & $41,5(12,28)$ & 70 & 25 & 5 & 45 & 55 & - & - \\
\hline Avós & 20 & $58,75(5,48)$ & 30 & 20 & 50 & 70 & 30 & - & - \\
\hline Professoras de creche & 20 & $35,5(9,81)$ & 5 & 50 & 45 & 70 & 30 & - & - \\
\hline
\end{tabular}


Questionário de dados sociodemográficos com informações sobre idade, escolaridade e o local de criação dos participantes; e (b) Escalas de Autonomia, Interdependência e Autonomiarelacionada de Kağitçibaşi (2007) em sua versão traduzida para o português e validada por Seidl-de-Moura, Ziviani, Fioravanti-Bastos e Carvalho (2013).

As escalas de Kağitçibaşi $(2007,2012)$ têm como objetivo avaliar as características de indivíduos conforme suas concepções de duas dimensões específicas: agência e distância interpessoal. Partindo dessas dimensões, empiricamente, três tendências de desenvolvimento do self são avaliadas: de autonomia, de relação e de autonomiarelacionada. Exemplos dos itens que compõem as três escalas são: item 13 (autonomia) - "Não gosto de interferência de nenhuma pessoa em minha vida, ainda que seja uma pessoa próxima a mim"; item 15 (relação) - (item invertido) "Não compartilho minhas questões pessoais com ninguém, mesmo que sejam pessoas muito próximas"; item 24 (autonomiarelacionada) - "Uma pessoa pode sentir-se tanto autônoma quanto ligada àqueles que lhe são íntimos".

\section{Procedimentos}

De coleta de dados. Após a aprovação do projeto pelo Comitê de Ética, os participantes foram convidados a responder ao questionário e assinaram o termo de consentimento livre e esclarecido.

O recrutamento dos adolescentes e jovens participantes do Estudo 1 foi realizado por meio de visitas a escolas de ensino fundamental e médio, assim como por indicação dos próprios pesquisadores. No Estudo 2, foram realizadas visitas a diversas creches na cidade do Rio de Janeiro e a famílias indicadas pela equipe de pesquisadores.

Em todos os casos, os sujeitos foram entrevistados em local de sua conveniência e preencheram individualmente os questionários. Houve auxílio dos pesquisadores para o preenchimento apenas quando solicitado.

De análise de dados. Para comparar os diferentes escores do mesmo participante e identificar qual dimensão (autonomia, interdependência, autonomia-relacionada) é mais valorizada pelos sujeitos da amostra, foram realizados testes $t$ pareados.
A ANOVA univariada foi utilizada para a comparação intergeracional da autonomia, interdependência e autonomiarelacionada (Estudo 1), para a comparação dos escores entre os diferentes perfis de cuidadoras (Estudo 2) e para avaliar o efeito do local de criação (dentro ou fora da capital do estado do Rio de Janeiro) sobre os escores de autonomia, interdependência e autonomia-relacionada dos participantes. A relação entre a idade dos participantes e seus escores de autonomia, autonomia-relacionada e interdependência foi analisada através de testes de correlação de Pearson. Por fim, para avaliar o efeito conjunto da idade, escolaridade e local de criação (variáveis explicativas) sobre os escores das diferentes cuidadoras do Estudo 2 (variáveis resposta), testou-se um modelo de regressão múltipla.

O modelo de regressão referente ao Estudo 1 considerou o escore do filho nas escalas de autonomia, interdependência e autonomia-relacionada como variável resposta e os escores dos pais nas mesmas escalas como variáveis explicativas. Acredita-se que esse processo seja mediado pelo nível de escolaridade, pelo local de criação dos participantes (dentro ou fora da capital do estado do RJ) e pela idade e, portanto, essas variáveis foram incluídas como covariadas de ajuste no modelo.

Todas as análises foram realizadas no software estatístico SPSS 20 e na plataforma $\mathrm{R}$ versão 3.0.2.

\section{Resultados e Discussão}

\section{Valorização da Autonomia, Autonomia-Relacionada e Interdependência / Relação}

Os resultados de testes t pareados indicaram que nos grupos dos Estudos 1 e 2, os escores de autonomia-relacionada foram os mais elevados para todos os participantes. Em ambas as amostras (Estudos 1 e 2), os escores de autonomia foram significativamente mais baixos que todos os outros escores.

Considerando apenas a comparação dos diferentes grupos de cuidadoras (Estudo 2), a ANOVA univariada indicou que as mães apresentaram escores significativamente mais altos de autonomia-relacionada do que as demais cuidadoras $(F(3,116)=3,02 ; p<0,05)$.

Tabela 2. Médias e Desvios Padrão dos escores de Autonomia, Interdependência e Autonomia Relacionada dos participantes dos Estudos 1 e 2

\begin{tabular}{|c|c|c|c|c|}
\hline & & $\begin{array}{l}\text { Média dos Escores de } \\
\text { Autonomia (DP) }\end{array}$ & $\begin{array}{l}\text { Média dos Escores de } \\
\text { Interdependência (DP) }\end{array}$ & $\begin{array}{c}\text { Média dos Escores de } \\
\text { Autonomia Relacionada (DP) }\end{array}$ \\
\hline \multirow[t]{4}{*}{ Estudo 1} & Avós/Avôs & $27,28(5,01)$ & $32,20(4,87)$ & $35,13(5,18)$ \\
\hline & Pais & $27,25(5,22)$ & $31,37(4,85)$ & $36,70(5,65)$ \\
\hline & Mães & $27,44(5,14)$ & $32,21(4,24)$ & $38,61(5,44)$ \\
\hline & Filhos & $26,50(4,34)$ & $33,39(4,60)$ & $40,32(4,62)$ \\
\hline \multirow[t]{4}{*}{ Estudo 2} & Mães & $29,48(5,10)$ & $32,72(5,05)$ & $40,78(4,61)$ \\
\hline & Babás & $29,05(4,94)$ & $30,85(4,13)$ & $36,55(5,15)$ \\
\hline & Avós & $27,95(6,19)$ & $33,70(3,96)$ & $36,80(6,06)$ \\
\hline & Professoras de Creche & $29,75(6,33)$ & $33,05(4,60)$ & $41,25(13,92)$ \\
\hline
\end{tabular}


Efeito da Escolaridade sobre os Escores de Autonomia, Autonomia-Relacionada e Interdependência

Houve uma diferença significativa no nível educacional dos participantes. Entre as famílias representadas pela amostra do Estudo 1, os filhos tiveram o maior nível educacional da família $\left(x_{6}^{2}=85,82 ; p<0,05\right)$. Foi possível identificar nessa amostra o padrão ascendente de escolarização da população brasileira ao longo dos anos, conforme reportado no último relatório da PNAD 2012 (Instituto Brasileiro de Geografia e Estatística [IBGE], 2012). Entre as cuidadoras de crianças pequenas, representadas pela amostra do Estudo 2, também houve diferença significativa no nível educacional $\left(x_{6}^{2}=64,37\right.$; $p<0,05)$. As mães tiveram o maior índice de escolarização da amostra e as babás o índice mais baixo.

Analisando o efeito da escolaridade sobre as dimensões de autonomia, interdependência e autonomia-relacionada, por meio da ANOVA univariada, entre os participantes do Estudo 1 , foi encontrado efeito significativo do nível de escolaridade sobre os escores de autonomia $(F(2,104)=4,41 ; p<0,05) \mathrm{e}$ autonomia-relacionada dos pais $(F(2,104)=4,76 ; p<0,05)$. Aqueles com maior nível de escolaridade apresentaram maior valorização da autonomia e autonomia-relacionada em comparação com pais de menor nível educacional. Para as mães, houve efeito significativo da escolaridade sobre os escores de interdependência $(F(2,104)=3,45 ; p<0,05)$ e autonomia-relacionada $(F(2,104)=11,51 ; p<0,05)$. Em ambos os casos, mães com escolaridade igual ou acima do nível superior incompleto apresentaram maiores escores em comparação a mães de escolaridade inferior. No grupo de filhos, a escolaridade apresentou o mesmo efeito do grupo de mães, afetando de forma semelhante os escores de interdependência $(F(2,104)=4,89 ; p<0,05)$ e autonomiarelacionada $(F(2,104)=6,61 ; p<0,05)$. Para os avôs/avós, foi identificado efeito da escolaridade apenas sobre os escores de autonomia-relacionada $(F(2,51)=8,57 ; p<0,05)$. Avôs e avós de maior nível educacional tiveram escores de autonomiarelacionada significativamente maiores que avôs e avós de nível de escolaridade inferior.

Esperava-se um efeito da escolaridade na valorização da autonomia, como foi observado em Seidl-de-Moura et al. (2008) em relação a tipos de metas de socialização. Isso ocorreu para os pais, mas não para as mães, filhos e avôs/avós.

Os resultados referentes à escolaridade e interdependência não foram na direção teoricamente esperada. Esperava-se que sujeitos com maior nível de escolarização apresentassem menores escores nessa escala. Para investigar se essa modificação de efeito estava relacionada com alguma das covariadas do estudo, estas foram testadas em interação com a escolaridade como preditoras da interdependência. Análises de regressão simples indicaram que o termo de interação escolaridade * local de criação é um preditor significativo da interdependência dos filhos e das mães. De posse desses resultados, estratificamos a amostra pela variável local de criação (criados na capital do Rio de Janeiro versus criados fora da capital do estado) e repetimos a ANOVA univariada da escolaridade sobre o escore de interdependência. Conforme esperado, o efeito positivo da escolaridade perdeu significância estatística para as mães criadas na capital $(F(2$,
$63)=1,55 ; p>0,05)$ e criadas fora da capital $(F(2,38)=3,09$; $p>0,05)$.

O local de criação também diluiu o efeito da escolaridade para os filhos, não houve efeito dessa variável na comparação intergrupal de escolaridade dos filhos criados na capital $(F(2$, $69)=2,95 ; p>.0,05)$ e criados fora da capital $(F(2,32)=2,24$; $p>0,05)$. Analisados em conjunto, esses resultados informam que, para as mães e filhos do Estudo 1, o efeito da escolaridade sobre a interdependência pôde ser em parte explicado pelo local em que esses participantes foram criados.

$\mathrm{Na}$ amostra do Estudo 2, foi encontrado efeito significativo da escolaridade apenas sobre o escore de autonomiarelacionada das mães $(F(2,59)=12,34 ; p<0,05)$. Mães com maior nível educacional apresentaram maior valorização da autonomia-relacionada. Considerando-se os resultados do efeito da escolaridade na autonomia-relacionada dos filhos no Estudo 1 e das mães no Estudo 2, pode-se pensar que essa tendência não é estática e sofre alterações de acordo com a experiência educacional. O efeito esperado da escolaridade sobre a autonomia em contextos de sociedades mais individualistas revelou-se, ao invés disso, sobre a autonomiarelacionada neste contexto específico brasileiro.

\section{Efeito do Local de Criação sobre os Escores de Autonomia, Interdependência e Autonomia- Relacionada}

A comparação das médias de autonomia, interdependência e autonomia-relacionada dos participantes que foram criados na capital do estado do Rio de Janeiro com participantes criados em outros municípios revelou diferenças significativas apenas entre os escores de interdependência dos filhos que foram criados dentro ou fora da capital do estado $(F(1,105)=6,44 ; p<0,05)$ e nos escores de autonomia das mães do Estudo $2(\mathrm{~F}(2,57)=3,61 ; p<0,05)$. Mães criadas na capital tiveram maiores escores de autonomia. Esse resultado é consistente com a literatura e as hipóteses dos efeitos da urbanização sobre crenças e valores de cuidadores (Seidlde-Moura et al., 2008).

\section{Efeito Conjunto das Variáveis nos Estudos 1 e 2}

Conforme relatado no início da seção de resultados, o escore de autonomia-relacionada foi o mais elevado entre os escores dos filhos (Estudo 1) e das mães (Estudo 2), caracterizando-se a autonomia-relacionada como tendência predominante desses grupos. Portanto, para testar a capacidade preditiva das variáveis abordadas neste estudo sobre o escore de autonomia-relacionada dos filhos (Estudo 1) e das mães (Estudo 2), foram ajustados quatro modelos de regressão linear múltipla.

Considerando o escore de autonomia-relacionada dos filhos como variável resposta, dois modelos foram testados. O Modelo 1, que incluía todas as variáveis explicativas, apresentou ajuste adequado $\left(R^{2}\right.$ ajustado $\left.=0,59 ; p<0,05\right)$. No entanto, devido ao grande número de variáveis explicativas e à presença de alguns valores extremos (outliers), os pressupostos de homocedasticidade e normalidade da variável 
Tabela 3. Coeficientes de regressão dos modelos preditivos para o Escore de Autonomia-relacionada dos Filhos (Estudo 1

\begin{tabular}{|c|c|c|c|c|}
\hline \multirow[t]{2}{*}{ Variáveis } & \multicolumn{2}{|r|}{ Modelo 1} & \multicolumn{2}{|c|}{ Modelo 2} \\
\hline & $\beta$ & (IC 95\%) & $\beta$ & (IC 95\%) \\
\hline \multicolumn{5}{|l|}{ Escore de Autonomia } \\
\hline Avô/Avó & $-0,0242$ & $(-0,2027 ; 0,1543)$ & & \\
\hline Pai & $0,2352 *$ & $(0,0104 ; 0,4601)$ & $0,2276^{* *}$ & $(0,0677 ; 0,3875)$ \\
\hline Mãe & $-0,1424$ & $(-0,4090 ; 0,1242)$ & $-0,2026 * *$ & $(-0,3578 ;-0,0473)$ \\
\hline \multicolumn{5}{|l|}{ Escore de Interdependência } \\
\hline Avô/Avó & 0,1277 & $(-0,1344 ; 0,3899)$ & & \\
\hline Pai & 0,0120 & $(-0,2041 ; 0,2279)$ & & \\
\hline Mãe & $-0,1069$ & $(-0,3769 ; 0,1632)$ & & \\
\hline \multicolumn{5}{|l|}{ Escore de Autonomia-relacionada } \\
\hline Avô/Avó & $0,3252 * *$ & $(0,1330 ; 0,5174)$ & 0,3384 & $(, 1766 ; 0,5000)$ \\
\hline Pai & $-0,0528$ & $(-0,2937 ; 0,1882)$ & & \\
\hline Mãe & 0,1369 & $(-0,1485 ; 0,4222)$ & $0,1995^{*}$ & $(-0,0083 ; 0,4073)$ \\
\hline \multicolumn{5}{|l|}{ Idade } \\
\hline Avô/Avó & 0,0077 & $(-0,1934 ; 0,2088)$ & & \\
\hline Pai & 0,1480 & $(-0,0901 ; 0,3865)$ & $0,1796^{*}$ & $(0,0041 ; 0,3551)$ \\
\hline Mãe & $-0,1703$ & $(-0,4386 ; 0,0980)$ & $-0,2002 *$ & $(-0,3687 ;-0,0317)$ \\
\hline Filho & $-0,0483$ & $(-0,5777 ; 0,4810)$ & & \\
\hline \multicolumn{5}{|l|}{ Escolaridadea } \\
\hline \multicolumn{5}{|l|}{ Avô/Avó } \\
\hline Médio completo & 1,6893 & $(-0,7748 ; 4,1534)$ & 1,4252 & $(-0,4691 ; 3,3195)$ \\
\hline Acima do superior incompleto & 1,6285 & $(-1,5306 ; 4,7876)$ & 1,7536 & $(-0,4566 ; 3,9638)$ \\
\hline \multicolumn{5}{|l|}{ Pai } \\
\hline Médio completo & $-1,2759$ & $(-3,8690 ; 1,3171)$ & $-1,2383$ & $(-3,0588 ; 0,5822)$ \\
\hline Acima do superior incompleto & $-2,4081$ & $(-5,1060 ; 0,2899)$ & $-1,8436^{*}$ & $(-3,8039 ; 0,1166)$ \\
\hline \multicolumn{5}{|l|}{ Mãe } \\
\hline Médio completo & 1,3830 & $(-1,6581 ; 4,4241)$ & 1,1778 & $(-0,7891 ; 3,1447)$ \\
\hline Acima do superior incompleto & 1,9944 & $(-0,5277 ; 4,5164)$ & $2,1692 *$ & $(0,2955 ; 4,0428)$ \\
\hline \multicolumn{5}{|l|}{ Filho } \\
\hline Médio completo & 0,2675 & $(-4,9245 ; 1,4578)$ & $-2,0240$ & $(-4,4883 ; 0,4402)$ \\
\hline Acima do superior incompleto & $-1,7334$ & $(-3,1289 ; 3,6640)$ & $-1,1574$ & $(-3,4377 ; 1,1230)$ \\
\hline \multicolumn{5}{|l|}{ Local de criaçãob } \\
\hline \multicolumn{5}{|l|}{ Avô/Avó } \\
\hline Capital RJ & $-0,0505$ & $(-0,1233 ; 0,0223)$ & $-5,0937 *$ & $(-10,3295 ; 0,1421)$ \\
\hline \multicolumn{5}{|l|}{ Pai } \\
\hline Capital RJ & 1,1338 & $(-1,4008 ; 3,6683)$ & & \\
\hline \multicolumn{5}{|l|}{ Mãe } \\
\hline Capital RJ & $-0,1456$ & $(-2,7826 ; 2,4915)$ & & \\
\hline \multicolumn{5}{|l|}{ Filho } \\
\hline Capital RJ & $-0,1946$ & $(-3,6758 ; 3,2866)$ & & \\
\hline \multicolumn{5}{|l|}{ Sexo do Filho } \\
\hline Feminino & $-0,0205$ & $(-2,5035 ; 2,4625)$ & & \\
\hline $\mathbf{R}^{2}$ & 0,68 & & 0,65 & \\
\hline $\mathbf{R}^{2}$ ajustado & 0,32 & & 0,48 & \\
\hline
\end{tabular}

Nota. $* \mathrm{p}<0,05 ; * * \mathrm{p}<0,01$; acategoria de referência $=$ até o ensino médio incompleto; bcategoria de referência= fora da capital do estado do Rio de Janeiro; Modelo 2= mesmas variáveis do modelo 1 submetidas ao método backward com critério de significância 95\% 
resposta não foram atendidos. O Modelo 2, que teve os outliers removidos (através do método da distância de Mahalanobis) e as variáveis selecionadas pelo método backward, apresentou melhor ajuste, indicando que as variáveis independentes selecionadas foram capazes de explicar $72 \%$ da variância da autonomia-relacionada dos filhos $\left(R^{2}\right.$ ajustado=0,72; $p<0,05)$. Além disso, os pressupostos de normalidade da variável resposta, homocedasticidade, normalidade dos erros e ausência de multicolinearidade foram verificados através do pacote gvlma do software estatístico $\mathrm{R}$, indicando a aprovação do Modelo 2 com base nesses critérios.

Conforme os coeficientes do Modelo 2, a idade do pai, a idade do filho e a autonomia-relacionada dos pais e das mães foram preditores positivos do escore de autonomiarelacionada dos filhos. Com efeito oposto, o aumento do escore de autonomia da mãe predisse uma menor valorização da autonomia-relacionada dos filhos. Diferenças de gênero foram relevantes para o modelo preditivo de autonomiarelacionada dos jovens neste estudo. Ser mulher predisse um aumento significativo da valorização da autonomiarelacionada em comparação com jovens do sexo masculino.

Tabela 4. Coeficientes de regressão do modelo preditivo para o Escore de Autonomia-relacionada das Mães (Estudo 2).

\begin{tabular}{lcl}
\hline Variáveis & $\boldsymbol{\beta}$ & $(\mathbf{I C ~ 9 5 \% )}$ \\
\hline Idade & $0,433^{* *}$ & $(0,215 ; 0,651)$ \\
Escolaridade $^{\mathrm{a}}$ & & \\
$\quad$ Médio completo & $5,0092^{*}$ & $(0,0788 ; 9,9396)$ \\
$\quad$ Acima do superior incompleto & $7,4166^{* *}$ & $(3,1649 ; 11,6683)$ \\
Local de criação & & \\
Capital RJ & 1,3730 & $(-1,1660 ; 3,9120)$ \\
$\mathbf{R}^{\mathbf{b}}$ & 0,45 & \\
$\mathbf{R}^{2}$ ajustado & 0,43 & \\
\hline
\end{tabular}

Nota. ${ }^{*} p<0,05 ; * * p<0,01 ;{ }^{a}$ categoria de referência $=$ até o ensino médio incompleto ${ }^{\mathrm{b}}$ categoria de referência $=$ fora da capital do estado do Rio de Janeiro

Esse efeito permaneceu o mesmo quando ajustado pelas outras covariadas do modelo (vide Tabela 3 ).

Esse modelo é, em geral, consistente com a literatura sobre as mudanças culturais e sociodemográficas e seus efeitos nos modelos de trajetórias de desenvolvimento (Kağitçibaşi, 2012). Além disso, contribui para a literatura ao focalizar o jovem, que foi a criança nessas trajetórias e o que prediz seu desfecho como uma pessoa com um perfil autônomo-relacionado. A autonomia e a escolaridade da mãe, a autonomia-relacionada da mãe e do pai, a idade do pai e a idade do próprio jovem, tais como medidos pelas escalas utilizadas, parecem afetar como esse filho vai se tornar. Parece haver, realmente, uma transmissão geracional de valores, balizada por características de idade e escolaridade dos pais.

O modelo preditivo para a autonomia-relacionada das mães (Estudo 2) foi estatisticamente significativo e apresentou um bom ajuste $\left(\mathrm{R}^{2}\right.$ ajustado $\left.=0,43 ; \mathrm{p}<0,05\right)$. $\mathrm{O}$ procedimento de verificação dos pressupostos para regressão linear múltipla foi realizado e as premissas foram atendidas. Conforme os coeficientes desse modelo (vide Tabela 4), o aumento da idade e da escolaridade das mães previu maior valorização da autonomia-relacionada. Não houve efeito significativo do local de criação.

\section{Considerações Finais}

Este estudo dá continuidade a uma série de trabalhos sobre trajetórias de desenvolvimento do self em contextos brasileiros que buscam investigar as formas assumidas na valorização da autonomia e da interdependência/relação com os outros, nesses contextos. Estudos anteriores concentraram-se em crenças maternas, em especial de mães de crianças pequenas. Apresentaram um perfil materno claro e consistente, que se repete em estudos com metodologias diversas e realizados em diferentes localidades do país (Seidlde-Moura, Carvalho et al., 2013).

Trata-se de um perfil que Kağitçibaşi (2012) denomina de self autônomo-relacionado. A autora argumenta favoravelmente à desejabilidade desse perfil em sociedades contemporâneas e discute os problemas de um perfil de valorização exacerbada da autonomia e independência (self autônomo-separado), característico de sociedades individualistas e pós-industriais em contextos urbanos. Por outro lado, discute ainda a impossibilidade de retorno a padrões de interdependência em sociedades rurais ou tradicionais que passaram por processos de modernização e desenvolvimento econômico (self heterônomo-relacionado). Desse modo, defende a meta de um modelo autônomorelacionado como uma trajetória de desenvolvimento saudável em contextos de mudança socioeconômica e cultural, como se vê em algumas sociedades na atualidade.

No presente estudo, buscou-se ampliar o foco de investigação, embora restringindo o contexto ao da cidade do Rio de Janeiro. Essa ampliação deu-se com a inclusão de outros grupos de participantes e etapas do ciclo vital. No Estudo 1, três gerações de famílias de jovens foram focalizadas: os filhos (jovens até 25 anos), seus pais e avós, além de suas mães. No Estudo 2, além das mães (que que já vinham sendo estudadas na literatura), foram ouvidas também outras cuidadoras de crianças pequenas (até um ano): babás, professoras de creche e avós

Este estudo apresenta limitações a serem consideradas em futuros trabalhos. Uma delas é a restrição a um contexto urbano de grande cidade, e outro é relativo aos instrumentos disponíveis e utilizados. Temos trabalhado com metodologias variadas, incluindo algumas escalas adaptadas para uso no Brasil. Neste trabalho, usamos as escalas de Kağitçibaşi (2007), mas a iniciativa de adaptação da escala revelou algumas limitações da mesma (Seidl-de-Moura, Ziviani et al., 2013), como a de dar conta das duas dimensões de agência e distância interpessoal. É necessário o esforço no desenvolvimento de novos instrumentos para estudar essas duas dimensões e sua tradução em perfis diferenciados de self. Esse é o objeto de nossos estudos atuais e, portanto, configura uma iniciativa que já está em andamento.

Apesar dessas limitações, e tendo em vista os resultados apresentados, consideramos que o trabalho contribui para a literatura sobre parentalidade, trajetórias de socialização e desenvolvimento em contextos, especialmente no que se refere ao seu foco principal de interesse, que foi o modelo 
de autonomia-relacionada. Os resultados seguiram, em geral, nas direções esperadas. Nesse sentido, foi identificada a valorização desse modelo por todos os participantes dos dois estudos, assim como sua dependência de variáveis sociodemográficas, como o nível educacional (Greenfield et al., 2008; Seidl-de-Moura, Carvalho et al., 2013). O efeito da escolaridade foi significativo sobre os escores de autonomia-relacionada dos pais, dos filhos e seus avós, assim como das mães participantes dos dois estudos (maior nível de escolaridade como preditor de maior valorização da autonomia-relacionada).

Não se tinha dados sobre babás, mas o estudo de Greenfield et al. (2008) nos levava a supor que apresentariam diferenças quando contrastadas com as mães. Não encontramos diferenças no perfil de autonomia-relacionada, identificado em todos os grupos. As professoras de creche, tal como em Friendlmeier et al. (2008), não diferiram das mães em sua valorização da autonomia e da relação. As avós apresentaram também o mesmo perfil das mães, diferentemente do observado no estudo de Ruela (2006), talvez porque, de forma diferente do estudo dessa autora, são de um contexto urbano de uma grande cidade.

A consistência familiar das trajetórias de socialização através das gerações ficou evidente nos resultados do Estudo 2. A presença de um modelo de autonomia-relacionada nos jovens participantes e seus pais e avós indica que o que foi observado nas amostras de mães (Seidl-de-Moura, Carvalho, et al., 2013) se aplica a outras faixas de idade.

\section{Referências}

Carvalho, R. V. C. C., Seidl-de-Moura, M. L, Vieira, M. L., \& Martins, G. (2014). Culture and developmental trajectories: A discussion on contemporary theoretical models. Early Child Development and Care, 184(11), 1559-1614. doi: 10.1080/03004430.2013.871273.

Friedlmeier, W., Schäfermeier, E., Vasconcellos, V. M. R., \& Trommsdorff, G. (2008). Self-construal and cultural orientation as predictors for developmental goals: A comparison between Brazilian and German caregivers. The European Journal of Developmental Psychology, 5, 39-67.

Greenfield, P. M., Flores, A., Davis, H., \& Salimkhan, G. (2008). What happens when parents and nannies come from different cultures? Comparing the caregiving belief systems of nannies and their employers. Journal of Applied Developmental Psychology, 29(4), 326-336.

Greenfield, P. M., Keller, H., Fuligni, A., \& Maynard, A. (2003). Cultural pathways through universal development. Annual Review of Psychology, 54, 461-490.

Instituto Brasileiro de Geografia e Estatística. (2012). População nos censos demográficos segundo as grandes regiões, as unidades da federação e a situação do domicílio (1960/2010). Retrieved from http://www.censo2010.ibge.gov.br/sinopse/ index.php?dados $=8 \& u f=00$
Kağitçibaşi, Ç. (2005). Autonomy and relatedness in cultural context: Implications for self and family. Journal of CrossCultural Psychology, 36, 403-422.

Kağitçibaşi, Ç. (1996). The autonomous-relational self: A new synthesis. European Psychologist,1(3), 180-186. doi: 10.1027/1016-9040.1.3.180

Kağitçibaşi, Ç. (2007). Family, self, and human development across cultures: Theory and applications. Mahwah, NJ: Lawrence Erlbaum Associates.

Kağitçibaşi, Ç. (2012). Socio-cultural change and integrative syntheses in human development: Autonomous-related self and social-cognitive competence. Child Development Perspectives, $6(1), 5-11$.

Keller, H. (2007). Cultures of infancy. Mahwah, NJ: Lawrence Erlbaum Associates.

Keller, H. (2012). Autonomy and relatedness revisited: Cultural manifestations of universal human needs. Child Development Perspectives, 6, 12-18.

Keller, H., Abels, M., Borke, J., Lamm, B., Lo, W., Su, Y. \& Wang, Y. (2007). Socialization environments of Chinese and Euro-American middle-class babies: Parenting behaviors, verbal discourses and ethnotheories. International Journal of Behavioral Development, 31(3), 210-217.

Keller, H., Kuensemueller, P., Abels, M., Voelker, S., Yovsi, R., Jensen, H., Papaligoura, Z., ...Mohite, P. (2005). Parenting, culture, and development: A comparative study. San José, CR: Instituto de Investigaciones Psicológicas.

Rapoport, A., \& Piccinini, C. A. (2004). A escolha do cuidado alternativo para o bebê e a criança pequena. Estudos de Psicologia (Natal), 9(3), 497-503.

Ruela, S. F. (2006). Um estudo intergeracional de crenças valorizadas por mães em uma comunidade rural do estado do Rio de Janeiro (Dissertação de Mestrado não publicada). Universidade do Estado do Rio de Janeiro, Rio de Janeiro, Rio de Janeiro, Brasil.

Seidl-de-Moura, M. L., Carvalho, R. V. C., \& Vieira, M. L. (2013). Brazilian mothers' cultural models: Socialization for autonomy and relatedness. Em M. L. Seidl-de-Moura (Ed.), Parenting in South American and African Contexts. Rijeka, Croácia: InTech.

Seidl-de-Moura, M. L., Lordelo, E., Vieira, M. L., Piccinnini, C. A., Siqueira, J. e O., Magalhães, C. M. C., ... Rimoli, A. (2008). Brazilian mothers' socialization goals: Intracultural differences in seven cities. International Journal of Behavioral Development, 32(6), 465-472.

Seidl-de-Moura, M. L., Targino, T., Nahum, K., Cruz, E. M., Amaral, G., \& De Marca, R. (2009). Parenting cultural models of a group of mothers from Rio de Janeiro, Brazil. Spanish Journal of Psychology, 12(2), 506-517.

Seidl-de-Moura, M. L., Mendes, D. M. L. F., Vieira, M. L., Korbarg, A. P. R., Pessôa, L. F., Bandeira, T. T. A (2013). Brazilian mothers description of their children and implicit cultural models. Psicologia: Teoria e Pesquisa, 29(3), 63-70.

Seidl-de-Moura, M. L., Ziviani, C., Fioravanti-Bastos, A. C., \& Carvalho, R. V. C. (2013). Adaptação brasileira das escalas de self autônomo, relacionado e autônomo-relacionado de Ç. Kağitçibaşi. Avaliação Psicológica, 12, 193-201. 
Vieira, M. L., Seidl-de-Moura, M. L., Lordelo, E., Piccinnini, C. A., Martins, G. D. F., Macarini, S. M., ... Rimoli, O. (2010). Brazilian mothers' beliefs about child-rearing practices. Journal of Cross-Cultural Psychology, 41(2), 195-211.
Vieira, M. L., Seidl-de-Moura, M. L., Macarini, S. M., Martins, G. D., Lordelo, E. da R., Tokumaru, R. S. (2010). Autonomy and interdependence: Beliefs of Brazilian mothers from State capitals and small towns. Spanish Journal of Psychology, 13, 816-824

Recebido em 28.07.2014

Primeira decisão editorial em 07.03.2016

Versão final em 29.03.2016

Aceito em 08.05.2016 